\title{
Stable periodic constant mean curvature surfaces and mesoscopic phase separation
}

\author{
AnTONio Ros ${ }^{\dagger}$ \\ Departamento de Geometría y Topología, \\ Facultad de Ciencias, Universidad de Granada, \\ 18071 Granada, Spain
}

[Received 5 June 2006 and in revised form 10 March 2007]

\begin{abstract}
We give a first comprehensive description of the stable solutions of the periodic isoperimetric problem in the case of lattice symmetry. This result is intended to elucidate the geometry of certain sophisticated interfaces appearing in mesoscale phase separation phenomena. We prove that closed, stable, constant mean curvature surfaces in $\mathbb{R}^{3} / \Gamma, \Gamma \subset \mathbb{R}^{3}$ being a discrete subgroup of translations with rank $k$, have genus $\leqslant k$. Finally, we extend the genus estimate to ambient spaces of the type $M \times \mathbb{R}$, where $M$ is a nonnegatively curved surface.
\end{abstract}

\section{Introduction}

Let $\Sigma$ be a compact two-sided surface with constant mean curvature in a flat 3-torus $T^{3}=\mathbb{R}^{3} / \Gamma$ obtained as the quotient of $\mathbb{R}^{3}$ by a lattice $\Gamma$. We say that $\Sigma$ is stable if it minimizes area up to second order for volume preserving deformations. This kind of surfaces appear, in particular, as solutions of the isoperimetric problem, which consists in describing area minimizing surfaces among those enclosing a given volume; see [23] for a general introduction to this topic. In this paper we will give a rather complete description of the geometry of closed, embedded, stable, constant mean curvature surfaces in a flat 3-torus. These surfaces may be used to model mesoscale phase separation phenomena in materials science (see [2, 3, 5, 9, 24] and references therein). As an example we can consider diblock copolymer melts. They are made up of two distinct polymer chains which are covalently bonded together. Under suitable conditions, the repulsive interaction between the different blocks causes the system to phase separate. The connectivity of the copolymer chains prevents macroscopic phase separation, and so block copolymer systems self-organize into periodic mesophases. Figure 1 contains the most frequent configurations appearing in this context: lamellar, body centered sphere packing, hexagonal cylindrical packing, perforated lamellar (with genus 2, modulo translations) and Schwarz $D$ surface and Schoen Gyroid $G$ (these last two surfaces have genus 3). Assuming the dominant term of the energy of the system is the area of the interface, the periodic isoperimetric problem is the simplest geometric model to explain the shape of these interfaces: we prescribe the periodicity and the volume fraction and we minimize the interfacial area. Note that the interfaces above match with the different items of the theorem below.

THEOREM 1 Let $\Sigma$ be a compact, orientable surface embedded with constant mean curvature in a flat 3-torus $T^{3}$, and $\widetilde{\Sigma} \subset \mathbb{R}^{3}$ its preimage in $\mathbb{R}^{3}$. If $\Sigma$ is stable, then it is one of the following:

(1) A finite collection of parallel planar 2-tori.

\footnotetext{
${ }^{\dagger}$ Partially supported by MEC-FEDER research projects MTM2004-02746. E-mail: aros@ugr.es
} 


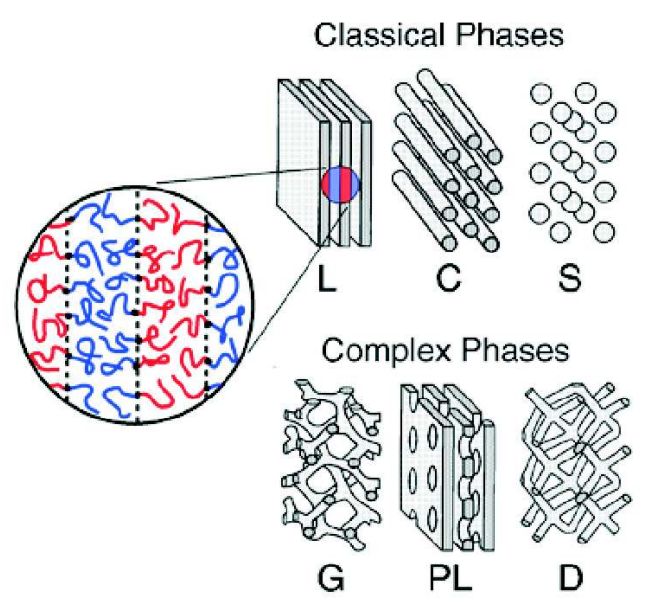

FIG. 1. Mesoscale phase separation in diblock copolymers [13].

(2) A round sphere.

(3) A (2-torus obtained as a quotient of a) circular cylinder.

(4) A connected surface of genus 2 such that the connected components of $\widetilde{\Sigma}$ are doubly periodic.

(5) A surface of genus 3 whose preimage $\widetilde{\Sigma}$ has triply periodic connected components.

Recall that a compact orientable surface is topologically equivalent to a 2 -sphere with finitely many handles and that the number of handles added is called the genus of the surface. Thus, the genus of the 2-sphere is zero and a 2-torus has genus one.

Theorem 1 will be proved as a consequence of Theorem 2 below. The structure of the space of stable surfaces of genus 2 and 3 still remains unclear. So, it could be useful to keep in mind the following tentative description.

CONJECTURE 1 Given a 3-torus $T^{3}$, the moduli space of closed, stable, embedded, constant mean curvature surfaces of genus 3 in $T^{3}$ with connected preimage in $\mathbb{R}^{3}$ can be naturally represented, if nonempty, by a connected, smooth convex arc in the (volume, area)-plane, which is symmetric with respect to the axis volume $=\operatorname{volume}\left(T^{3}\right) / 2$.

The hard part of the above statement is the connectedness of the moduli space. In the case of the simple cubic torus, the conjectural shape of this moduli space is given in Figure 2 Conjecture 1 extends to stable surfaces of genus 2 . In this case the moduli space should be described by a connected convex curve in the (volume, area)-plane that projects injectively on both the volume and the area axes.

Among self-assembled materials, mesoscopic wetting phenomena are particularly related to doubly periodic, stable, constant mean curvature surfaces (see [12]): under suitable conditions, a thin layer of liquid wetting a hydrophobic planar surface produces a pattern as in Figure 3 a periodic array of dry spots. As doubly periodic constant mean curvature surfaces always have a horizontal mirror symmetry (assuming the prescribed translations are horizontal), it follows that the corresponding periodic isoperimetric problems in $\mathbb{R}^{3}$ and in the halfspace $\left\{x_{3} \geqslant 0\right\}$ are equivalent. As another consequence of Theorem 2 below, we conclude that the assumptions 


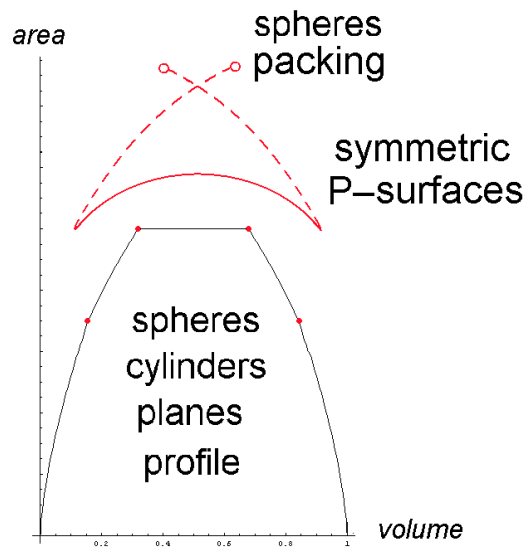

FIG. 2. Conjectural isoperimetric profile (at the bottom) of the simple cubic 3-torus $\mathbb{R}^{3} / \mathbb{Z}^{3}$ : spheres for small and large volumes, planes for middle values and cylinders for some range between them. According to Surface Evolver experiments [22], the isoperimetric problem in other 3-tori, like the face centered cubic torus, should admit solutions of genus 2 . The curve at the top represents the moduli space of the most symmetric constant mean curvature Schwarz $P$ surfaces in $\mathbb{R}^{3} / \mathbb{Z}^{3}$, as computed numerically in [2]. The dashed arcs correspond to unstable surfaces. Stable surfaces should consist of a symmetric subarc of the continuous red arc. Ross [27] has proved that the minimal $P$ surface, enclosing a volume equal to $1 / 2$, is stable.

(i) the pattern is doubly periodic,

(ii) the volume fraction of liquid is given,

(iii) the energy of the system is just the area (per unit cell) of the liquid surface,

predict the right experimental wetting phases: after reflection in the horizontal plane we obtain either round spheres, horizontal right cylinders, or constant mean curvature doubly periodic surfaces with genus 2 (modulo translations), like those in Figure 3.
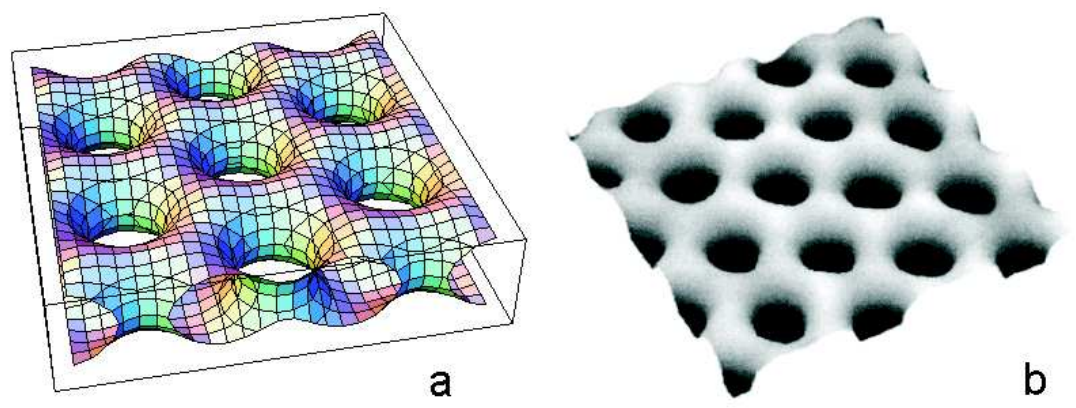

FIG. 3. (a) Doubly periodic surface with constant mean curvature and genus 2 (modulo translations). These surfaces were first constructed by Lawson [11] as conjugate surfaces of minimal surfaces in the 3-sphere. (b) Periodic pattern formed by a thin layer of liquid on a planar surface, [12]: this self-assembled wetting phenomenon may be modeled by stable doubly periodic constant mean curvature surfaces.

Let $\Gamma \subset \mathbb{R}^{3}$ be a discrete subgroup of translations with $\operatorname{rank}(\Gamma)=k$ and $\Sigma$ a compact orientable surface with constant mean curvature in the flat 3-manifold $\mathbb{R}^{3} / \Gamma$. If $k=0$, then Barbosa and do 
Carmo [4] proved that the only stable surfaces are round spheres. For $1 \leqslant k \leqslant 3$ it is known that, if $\Sigma$ is stable, then it must be either a union of planar surfaces or a connected surface of genus smaller than or equal to 3 (Ros [25]). This result is sharp for $k=3$ as Ross [27] has proved that the classical Schwarz $P$ minimal surface (of genus 3 ) in the simple cubic torus $\mathbb{R}^{3} / \mathbb{Z}^{3}$ is stable.

The next theorem improves the estimate of the genus of stable surfaces when $k=1$ and 2 . We will obtain the sharp result genus $(\Sigma) \leqslant k$.

THEOREM 2 Let $\Sigma$ be a compact two-sided surface with constant mean curvature immersed in the flat 3-manifold $\mathbb{R}^{3} / \Gamma, \Gamma$ being a rank $k$ discrete subgroup of translations of $\mathbb{R}^{3}, 0 \leqslant k \leqslant 3$. If $\Sigma$ is stable, then it consists of either a finite family of planar surfaces, or a connected surface of genus less than or equal to $k$.

Moreover, in the latter case, if genus $(\Sigma)=0$ or 1 , then $\Sigma$ is either a round sphere or (the quotient of) a right circular cylinder, respectively.

Then it follows from Ritore and Ros [21] that only a compact family in the moduli space of rank $k$ groups of translations $\Gamma$ admit embedded, closed, stable, constant mean curvature surfaces of genus $k=2$, 3. For $k=2$, Hauswirth, Pérez, Romon and Ros [9] describe an explicit small region in this moduli space, such that outside this region, the solutions of the isoperimetric problem in $\mathbb{R}^{3} / \Gamma$ consist just of spheres, cylinders and planar slabs.
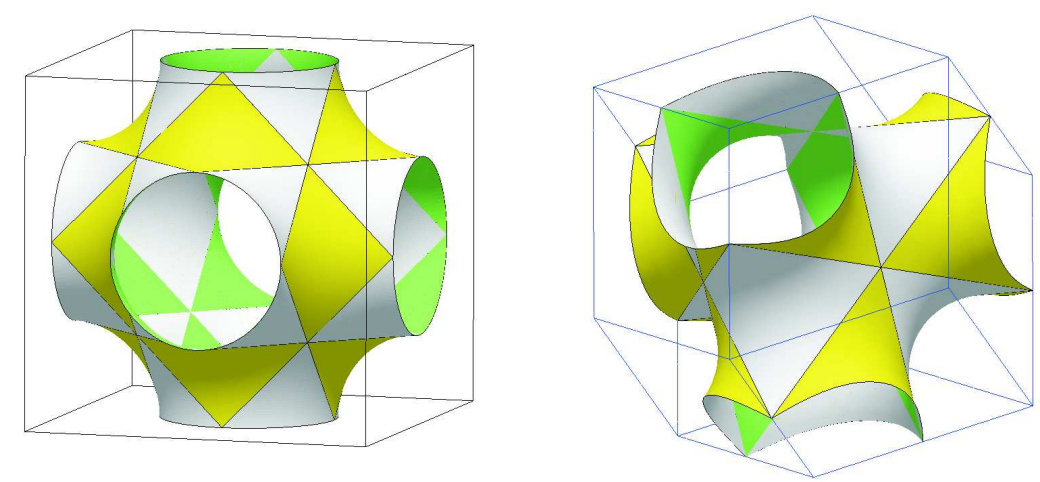

FIG. 4. The Schwarz $P$ surface (left) is the simplest triply periodic minimal surface. If we identify the opposite faces of the cube, we obtain a closed surface of genus three in the simple cubic 3-torus. The Schwarz $D$ surface (right) is a periodic minimal surface which induces a genus three surface in the face centered cubic 3-torus obtained by identifying the opposite faces of the rhombic dodecahedron. Both surfaces are stable, [27].

Theorem 2 is sharp in the cases $k=0,1$ and 3: The first two can be checked directly, and when $k=3$ Ross [27] proved that the minimal, genus 3 surfaces $P$ and $D$ of Schwarz and $G$ of Schoen are stable in the cubic 3-tori sc, fcc and bcc, respectively. It seems likely that the theorem is also sharp for $k=2$, that is, there should be stable surfaces of genus 2: In fact, by using Brakke's Surface Evolver, Romon [22] concludes that, if we take $\Gamma$ to be the hexagonal planar lattice, there should exist an isoperimetric surface of genus 2 in $\mathbb{R}^{3} / \Gamma$, as in Figure $3 \mathrm{a}$. This surface encloses the same volume as a circular cylinder of area equal to 2 area $\left(\mathbb{R}^{2} / \Gamma\right)$, but its area beats the area of the cylinder by a factor $=0.9998$. In case $\Gamma$ is a square planar lattice, the same kind of genus 2 stable constant mean curvature surface can be obtained by Evolver, but now its area is $\sim 2 \operatorname{area}\left(\mathbb{R}^{2} / \Gamma\right) \times 1.0003$. 
Finally, we will prove a general genus estimate for stable surfaces when the ambient space is the product of a nonnegatively curved surface and the real line. It is worth noting that the proof of the general case does not work in the flat situation considered above.

THEOREM 3 Let $M$ be a complete, orientable surface with nonnegative Gaussian curvature and $\Sigma$ a compact, orientable surface embedded in $M \times \mathbb{R}$ with constant mean curvature. If $\Sigma$ is stable, then either $M$ is compact and $\Sigma$ is a finite union of horizontal slices, or $\Sigma$ is a connected surface with genus $(\Sigma) \leqslant 2$.

It was proved in [25] that the genus of a closed stable surface in a 3-manifold with nonnegative Ricci curvature is at most 3. Pedrosa [17] solved the isoperimetric problem in the product $S^{2} \times \mathbb{R}$ of a round sphere and a line.

When we study stability problems, we need to construct test functions with controlled energy. In this paper we will first use test functions obtained from harmonic 1-forms on the surface (see Palmer [16] and Ros [25] for other applications of harmonic forms to stability problems). We will also construct test functions by using conformal maps between suitable pieces of $\Sigma$ and the 2sphere. Conformal maps have been used in eigenvalue estimates by Szegö [28], Hersch [10], Yang and Yau [29] and other authors.

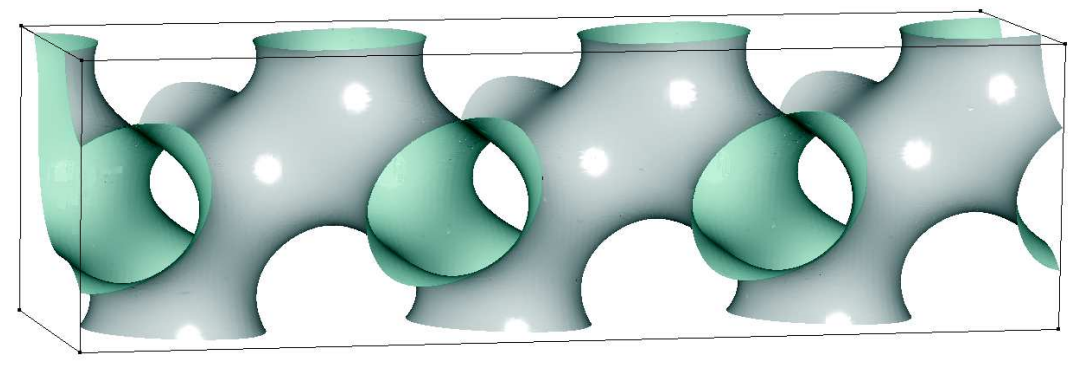

FIG. 5. The $G$ surface of A. Schoen is a triply periodic minimal surface which induces a closed stable surface of genus three in the body centered cubic 3-torus. It has screw axes of order four parallel to the edges of the cube, [24].

\section{Preliminaries}

Let $\Sigma$ be a compact surface immersed with constant mean curvature $H$ in an orientable Riemannian 3-manifold $\widetilde{M}$. We will assume along this paper that $\Sigma$ is two-sided, i.e. the unit normal vector $N$ of the immersion is globally well-defined; in our context this is equivalent to the orientability of $\Sigma$ itself. The surface $\Sigma$ is said to be stable if the second variation formula of the area is nonnegative for any volume preserving infinitesimal deformation. We consider on $\Sigma$ the quadratic Jacobi form

$$
Q(u, v)=\int_{\Sigma}\langle\nabla u, \nabla v\rangle-\left(|\sigma|^{2}+\operatorname{Ric}(N)\right) u v=-\int_{\Sigma} u L v, \quad u, v \in C^{\infty}(\Sigma),
$$

$\sigma$ being the second fundamental form of the embedding, $\operatorname{Ric}(N)$ the Ricci curvature of $\widetilde{M}$ in the normal direction $N$, and $L=\Delta+|\sigma|^{2}+\operatorname{Ric}(N)$ the Jacobi operator of the surface. The stability 
condition is equivalent to

$$
Q(u, u) \geqslant 0 \quad \text { for any } u \text { with } \int_{\Sigma} u=0
$$

(see [4]). Solutions of the isoperimetric problems, i.e. those closed surfaces in $\widetilde{M}$ which have least

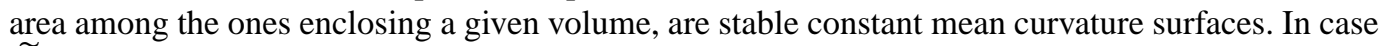
$\widetilde{M}$ is compact or even compact modulo symmetries, these surfaces are known to exist and to be smooth everywhere (see Morgan [15] and Gonzalez, Massari and Tamanini [7]). It is worth stating that the regularity for the isoperimetric problem pertains to local (in space) minimizers as well and not just to the global ones.

Along this paper we will assume that $\tilde{M}$ has nonnegative Ricci curvature Ric $\geqslant 0$. If $\Sigma$ is stable and disconnected, then it admits a nonvanishing locally constant function $u$ with mean value zero. Thus, it follows from (1) that $\Sigma$ is totally geodesic. If $\Sigma$ is connected, then Ros [25] proves that the genus of $\Sigma$ is $\leqslant 3$ (for earlier partial results see [6, 20, 19, 30]).

If $\widetilde{M}=M \times \mathbb{R}, M$ being an orientable surface, then the only closed minimal surfaces are unions of horizontal slices. For any real $c$, we have the reflection symmetry $(p, t) \mapsto(p, 2 c-t)$ with respect to the slice $t=c$. If $\Sigma$ is a compact embedded constant mean curvature surface in $M \times \mathbb{R}$ with $H \neq 0$, then from the Aleksandrov reflection technique [1] we conclude that (up to a vertical translation if necessary) $\Sigma$ is symmetric with respect to the slice $t=0$. Let us denote this mirror symmetry by $\tau: \Sigma \rightarrow \Sigma$. Moreover $\Sigma \cap\{t=0\} \neq \emptyset$ and $\Sigma_{+}=\Sigma \cap\{t \geqslant 0\}$ is a graph over a compact smooth region in $M$ with nonempty boundary.

Now we specialize to the case of periodic surfaces. If $\Gamma \subset \mathbb{R}^{3}$ is a discrete group of translations of rank $k$, i.e. $\Gamma$ is generated by $k$ linearly independent vectors, then the flat 3 -manifold $\mathbb{R}^{3} / \Gamma$ is isometric to $T^{k} \times \mathbb{R}^{3-k}$, where $T^{n}$ is an $n$-dimensional flat torus. A connected nonplanar properly embedded constant mean curvature surface $\Sigma \subset \mathbb{R}^{3}$ is said to be $k$-periodic if it admits a translation symmetry group $\Gamma$ of rank $k$ such that $\Sigma / \Gamma$ is compact. If the surface is compact $(k=0)$, then Aleksandrov [1] proved that it must be a round sphere. If $\Sigma$ is singly periodic $(k=1)$, Aleksandrov's technique gives that it must be a Delaunay surface of revolution. If moreover the quotient surface $\Sigma / \Gamma \subset \mathbb{R}^{3} / \Gamma$ is stable, then Ritoré and Ros [20] prove that $\Sigma$ is a right circular cylinder. Doubly periodic constant mean curvature surfaces $(k=2)$ were first constructed by Lawson [11]. If the surface is nonplanar, then $H \neq 0$, the genus of the quotient surface $\Sigma / \Gamma$ is at least 2, and Aleksandrov's technique provides a mirror plane parallel to $\Gamma$ dividing the surface into two graphical pieces. In Figure 3 a we can see a surface of this type, with $\Gamma$ the hexagonal planar lattice. For further examples see [18, 8], and for global properties of these surfaces and related ones see [26]. Finally, we notice that Aleksandrov's technique does not apply to the triply periodic case.

\section{The flat case}

Proof of Theorem 2 Assume that $\Sigma$ is not a collection of planar surfaces. In particular, it must be connected. Let $e_{1}, e_{2}$ be a positively oriented orthonormal basis of tangent vectors, $A$ the shape operator of $\Sigma$, and $K$ and $H$ its Gauss and mean curvatures, respectively. Thus $\left\langle A e_{i}, e_{j}\right\rangle=$ $\sigma\left(e_{i}, e_{j}\right), A^{2}=2 H A-K I$, where $I$ is the identity matrix, and $|\sigma|^{2}=4 H^{2}-2 K$.

Let $H^{1}(\Sigma)$ be the space of harmonic 1-forms. Recall that a 1-form $\omega$ on $\Sigma$ is harmonic if and only if it is closed, i.e. $\nabla \omega(x, y)=\nabla \omega(y, x)$ for all tangent vectors $x, y$ ( $\nabla \omega$ being the covariant derivative of $\omega$ ), and coclosed, that is, $\operatorname{div}(\omega)=0$ (the divergence operator being 
defined as $\left.\operatorname{div}(\omega)=\nabla \omega\left(e_{1}, e_{1}\right)+\nabla \omega\left(e_{2}, e_{2}\right)\right)$. Let us also recall that harmonic 1-forms $\omega$ satisfy the differential equation $\Delta \omega-K \omega=0$, where $\Delta$ denotes the rough Laplacian $(\Delta \omega)(v)=$ $\nabla^{2} \omega\left(e_{1}, e_{1}, v\right)+\nabla^{2} \omega\left(e_{2}, e_{2}, v\right)$. The Hodge star operator transforms a 1-form $\omega$ into another $\omega^{*}$ defined by $\omega^{*}\left(e_{1}\right)=\omega\left(e_{2}\right)$ and $\omega^{*}\left(e_{2}\right)=-\omega\left(e_{1}\right)$. If $g$ denotes the genus of the surface, then the space of harmonic 1-forms $H^{1}(\Sigma)$ has dimension $2 g$ and the Hodge star operator defines a natural anti-involution on $H^{1}(\Sigma), \omega \mapsto \omega^{*}$. Although the linear coordinates $x_{n}, n=1,2,3$, are multivalued on $\Sigma$, their differentials $\alpha_{n}=d x_{n}$ are well-defined on $\Sigma$ and satisfy

$$
\left(\Delta \alpha_{n}\right)(v)=-\alpha_{n}\left(A^{2} v\right) .
$$

If $\omega \in H^{1}(\Sigma)$, then we can consider the dual tangent vector field on $\Sigma$, viewed as an $\mathbb{R}^{3}$-valued function, $X=X_{\omega}: \Sigma \rightarrow \mathbb{R}^{3}$,

$$
X=\left(\left\langle\omega, \alpha_{1}\right\rangle,\left\langle\omega, \alpha_{2}\right\rangle,\left\langle\omega, \alpha_{3}\right\rangle\right) .
$$

Palmer [16] used the vector field $X$ to study the index of harmonic Gauss maps. By direct computation we obtain

$$
\begin{aligned}
\Delta X= & \left(\left\langle\Delta \omega, \alpha_{1}\right\rangle,\left\langle\Delta \omega, \alpha_{2}\right\rangle,\left\langle\Delta \omega, \alpha_{3}\right\rangle\right) \\
& +\left(\left\langle\omega, \Delta \alpha_{1}\right\rangle,\left\langle\omega, \Delta \alpha_{2}\right\rangle,\left\langle\omega, \Delta \alpha_{3}\right\rangle\right)+2\left(\left\langle\nabla \omega, \nabla \alpha_{1}\right\rangle,\left\langle\nabla \omega, \nabla \alpha_{2}\right\rangle,\left\langle\nabla \omega, \nabla \alpha_{3}\right\rangle\right) \\
= & K X-A^{2} X+2\langle\nabla \omega, \sigma\rangle N=2 K X-2 H A X+2\langle\nabla \omega, \sigma\rangle N,
\end{aligned}
$$

and therefore

$$
\Delta X+|\sigma|^{2} X=4 H^{2} X-2 H A X+2\langle\nabla \omega, \sigma\rangle N .
$$

By applying the stability quadratic form $Q$ to (the linear coordinates of) $X=X_{\omega}$ we have

$$
Q(X, X)=-\int_{\Sigma}\left\langle\Delta X+|\sigma|^{2} X, X\right\rangle=-\int_{\Sigma}\left(4 H^{2}|X|^{2}-2 H \sigma(X, X)\right) .
$$

If we denote by $X^{*}=X_{\omega^{*}}$ the dual tangent vector field of $\omega^{*}$, using the identities $\left|X^{*}\right|=|X|$ and $\left\langle X, X^{*}\right\rangle=0$, we conclude as in [16] that

$$
Q(X, X)+Q\left(X^{*}, X^{*}\right)=-\int_{\Sigma}\left(8 H^{2}|X|^{2}-4 H^{2}|X|^{2}\right)=-4 H^{2} \int_{\Sigma}|X|^{2} .
$$

We consider the map $f: H^{1}(\Sigma) \rightarrow \mathbb{R}^{3} \times \mathbb{R}^{3}$ given by

$$
f(\omega)=\left(\int_{\Sigma} X_{\omega}, \int_{\Sigma} X_{\omega^{*}}\right) .
$$

If $\operatorname{rank}(\Gamma)=k$, then there are $3-k$ linear coordinate functions $x_{n}$ globally well-defined on $\Sigma$. As harmonic 1-forms are closed and coclosed, the divergence of the 1-form $x_{n} \omega$ is

$$
\operatorname{div}\left(x_{n} \omega\right)=\left\langle\omega, d x_{n}\right\rangle+x_{n} \operatorname{div} \omega=\left\langle\omega, d x_{n}\right\rangle,
$$

and the divergence theorem will imply that $2(3-k)$ of the linear coordinates of $f(\omega)$ vanish. Hence, the dimension of the kernel of the linear map $f$ is at least $2 g-6+2(3-k)=2(g-k)$. If $g>k$, then $\operatorname{dim} \operatorname{Ker}(f)>0$ and there exists a nonzero harmonic form $\omega$ such that $f(\omega)=0$, that is, both $X_{\omega}$ 
and $X_{\omega^{*}}$ have mean value zero. From the stability hypothesis and $\sqrt{3}$ we obtain $0 \leqslant-H^{2} \int|X|^{2}$, and so $\Sigma$ must be a minimal surface and $Q(X, X)=Q\left(X^{*}, X^{*}\right)=0$. Hence, combining (1) with linear elliptic theory, we get vectors $a, a^{*} \in \mathbb{R}^{3}$ such that

$$
\Delta X+|\sigma|^{2} X=a, \quad \Delta X^{*}+|\sigma|^{2} X^{*}=a^{*} .
$$

On the other hand, if we put $H=0$ in 2 we have

$$
\Delta X+|\sigma|^{2} X=2\langle\nabla \omega, \sigma\rangle N, \quad \Delta X^{*}+|\sigma|^{2} X^{*}=2\left\langle\nabla \omega^{*}, \sigma\right\rangle N .
$$

As the normal direction along a nonplanar minimal surface cannot be constant, even locally, we deduce that $a=a^{*}=0$ and $\langle\nabla \omega, \sigma\rangle=\left\langle\nabla \omega^{*}, \sigma\right\rangle=0$, which is the same as saying that $\langle\nabla \omega, \sigma\rangle=$ $\left\langle\nabla \omega, \sigma^{*}\right\rangle=0$, where $\sigma^{*}$ is the second fundamental form of the conjugate minimal surface. Finally, we observe that, by harmonicity, $\nabla \omega$ is a symmetric traceless 2-tensor. As this kind of matrices form a vector plane, $\sigma$ and $\sigma^{*}$ generate this plane at the nonflat points of $\Sigma$. This would imply that $\omega$ should be a parallel 1-form, $\nabla \omega=0$, which exists only on flat surfaces. This contradiction proves that $g \leqslant k$, as claimed.

The result proved above is sharp for any $k=0,1,2$ and 3. If $\Gamma=0$ (i.e. $k=0$ ) the theorem says that $g=0$ and thus $\Sigma$ must be a round sphere by a classic result of Hopf. This gives a new proof of the Barbosa and do Carmo characterization of the sphere as the only closed stable constant mean curvature surface in $\mathbb{R}^{3}$ (see [4]).

If $\operatorname{rank}(\Gamma)=1$ (i.e. $\widetilde{M}=S^{1} \times \mathbb{R}^{2}$ ), the theorem allows us to conclude that $g=0$ or 1 and therefore $\Sigma$ is either a round sphere or a right cylinder, as follows from Ritoré and Ros [20]. We remark that this was previously known only in the embedded case.

The main novelty in Theorem 2 is the estimate genus $(\Sigma) \leqslant 2$ in the case $\operatorname{rank}(\Gamma)=2$. A stable surface of genus 2 in $\mathbb{R}^{3} / \Gamma$, where $\Gamma$ is the hexagonal 2-lattice, will look like the surface in Figure 3 a.

If $\Gamma$ is a 3-lattice (i.e. $k=3$ ) the above gives a new proof of the fact that closed stable constant mean curvature surfaces in a flat 3 -torus have genus $\leqslant 3$ (Ros [25]). However, this result can be qualitatively improved in the embedded case, by combining all the power of Theorem 2 with topological properties of constant mean curvature embeddings. This improvement is given in Theorem 1 and describes exhaustively all the different geometries of stable solutions of the periodic isoperimetric problem, when the prescribed symmetry group is a lattice.

Proof of Theorem 1 Assume that $\Sigma$ is a nonplanar constant mean curvature stable surface in a flat 3-torus $T^{3}$. Then Theorem 2 implies that $\Sigma$ is a connected surface of genus $g \leqslant 3$.

Moreover the constant mean curvature assumption implies that $\Sigma$ encloses a mean convex region (see [21]) which has the topology of a solid doughnut of genus $g$ (see Meeks [14]). In particular, the surfaces lift to a $k$-periodic surface in $\mathbb{R}^{3}$, with $k \leqslant g$.

If $g=0$, the surface lifts to an embedded closed surface in $\mathbb{R}^{3}$ with constant mean curvature and Aleksandrov's theorem [1] implies that $\Sigma$ is a round sphere.

In the case $g=1$, Ritore and Ros [20] proved that stable surfaces are quotients of right circular cylinders.

If $g=2$, then the connected components of the preimage $\widetilde{\Sigma} \subset \mathbb{R}^{3}$ of $\Sigma$ are at most doubly periodic. The nonperiodic and the singly periodic possibilities are discarded by using Aleksandrov's technique [1]. So we obtain assertion (2) of the theorem. 
Finally, if we suppose that $g=3$, then as before we deduce that the components of $\widetilde{\Sigma}$ are not $k$ periodic, for $k=0,1$. If these components were doubly periodic, then there would exist an infinite covering $\mathbb{R}^{3} / \Gamma \rightarrow T^{3}$, for a certain group of translations $\Gamma$ of rank 2 , such that the embedding of $\Sigma$ in $T^{3}$ lifts to a stable constant mean curvature embedding of $\Sigma$ in $\mathbb{R}^{3} / \Gamma$. As this contradicts Theorem 2, we conclude that the connected components of $\widetilde{\Sigma}$ are triply periodic, as claimed.

\section{The case of nonnegative curvature}

Let $\Sigma$ be a stable constant mean curvature surface in $M \times \mathbb{R}$, where $M$ is a nonnegatively curved surface. If $\Sigma$ is either disconnected or a minimal surface, then we know that it consists of a union of horizontal slices. So, henceforth we will assume that $\Sigma$ is connected and $H \neq 0$. Recall that this implies that $\Sigma$ admits the mirror symmetry $\tau:(p, t) \mapsto(p,-t)$ and that $\Sigma_{+}=\Sigma \cap\{t \geqslant 0\}$ projects injectively on $t=0$ and has nonempty boundary.

The following statement can be proved by using an argument of Yang and Yau [29].

LEMMA 4 Let $\phi: \Sigma \rightarrow S^{2}$ be a continuous map from a compact surface into the unit sphere. If the preimage $\phi^{-1}(x)$ of any point $x \in S^{2}$ is of measure zero, then there is a conformal transformation of the sphere $g: S^{2} \rightarrow S^{2}$ such that the vector-valued map $g \circ \phi: \Sigma \rightarrow S^{2} \subset \mathbb{R}^{3}$ has mean value zero,

$$
\int_{\Sigma} g \circ \phi \mathrm{d} A=0
$$

Proof of Theorem 3 First observe that, as $M$ has nonnegative curvature, it must be homeomorphic either to the sphere $S^{2}$, the plane $\mathbb{R}^{2}$, the cylinder $S^{1} \times \mathbb{R}$, or the torus $T^{2}$. Moreover in the last two cases $M$ must be flat. Let us denote by $g$ the genus of $\Sigma$. We discuss the following possibilities separately.

CASE 1: $M$ is a spherical domain. As $\Sigma_{+}$is homeomorphic to a region in $M$, we see that $\Sigma_{+}$ admits a conformal injective map into the unit 2 -sphere,

$$
\phi_{+}: \Sigma_{+} \rightarrow S^{2} \subset \mathbb{R}^{3} .
$$

We extend $\phi_{+}$to a symmetric piecewise smooth map $\phi: \Sigma \rightarrow S^{2}$ so that $\phi=\phi_{+}$on $\Sigma_{+}$and $\phi \circ \tau=\phi$. Moreover, from Lemma 4 we can assume that $\phi$ has mean value zero in $\mathbb{R}^{3}$,

$$
\int_{\Sigma} \phi \mathrm{d} A=0
$$

Hence the linear coordinates of $\phi=\left(\phi_{1}, \phi_{2}, \phi_{3}\right)$ are admissible test functions for the stability condition (11) and in this way we obtain

$$
0 \leqslant \sum_{n} Q\left(\phi_{n}, \phi_{n}\right)=\int_{\Sigma}\left(|\nabla \phi|^{2}-\left(\operatorname{Ric}\left(e_{1}\right)+\operatorname{Ric}\left(e_{2}\right)+4 H^{2}-2 K\right)|\phi|^{2}\right),
$$

where we have used the fact that $\operatorname{Ric}(N)+|\sigma|^{2}=\operatorname{Ric}\left(e_{1}\right)+\operatorname{Ric}\left(e_{2}\right)+4 H^{2}-2 K$, which follows from the Gauss equation. As the Ricci curvature of $M \times \mathbb{R}$ is nonnegative, applying the fact that $\phi$ is symmetric, $|\phi|=1$ and that, $\phi_{+}$being conformal, its energy is twice the area of its spherical image $\phi\left(\Sigma_{+}\right)$, the above inequality transforms into

$$
0 \leqslant 4 \operatorname{area}\left(\phi\left(\Sigma_{+}\right)\right)-\int_{\Sigma}\left(4 H^{2}-2 K\right)<4 \operatorname{area}\left(S^{2}\right)-4 H^{2} \operatorname{area}(\Sigma)+4 \pi(2-2 g) .
$$


In the last inequality we have used the Gauss-Bonnet theorem $\int_{\Sigma} K=2 \pi(2-2 g)$ and the fact that the area of $\phi\left(\Sigma_{+}\right)$is smaller than area $\left(S^{2}\right)=4 \pi$. So, finally we obtain

$$
H^{2} \operatorname{area}(\Sigma)<2 \pi(3-g)
$$

which implies $g \leqslant 2$.

CASE 2: $M$ is a 2-torus. We simply note that in this case $M$ should be a flat torus and so the result follows from Theorem 2 .

It is not clear if the hypothesis on the embeddedness of $\Sigma$ in Theorem 3 may be removed or even relaxed to Aleksandrov embeddedness. Although Aleksandrov embedded surfaces admit a reflection symmetry, a priori, the genus of $\Sigma_{+}$need not be bounded under this weaker assumption.

\section{REFERENCES}

1. Aleksandrov, A. D. Uniqueness theorems for surfaces in the large I. Vestnik Leningrad. Univ. 11 (1956), 5-17 (in Russian). MR 0086338

2. Anderson, D. M., Davis, H. T., Nitsche, J. C. C., \& Scriven, L. E. Periodic surfaces of prescribed mean curvature. Adv. Chem. Phys. 77 (1990), 337-396.

3. Anderson, D. M., Hoffman, D., Henkee, C. S., \& Thomas, E. L. Periodic area-minimizing surfaces in block copolymers. Nature 334 (1988), 598-602.

4. BarbosA, J. L., \& DO CARMo, M. Stability of hypersurfaces with constant mean curvature. Math. Z. 185 (1984), 339-353. Zbl 0513.53002 MR 0735120

5. Choksi, R., \& Sternberg, P. Periodic phase separation: the periodic isoperimetric and Cahn-Hilliard problems. Interfaces Free Bound. 8 (2006), 371-392. Zbl 1109.35092 MR 2273234

6. El Soufi, A., \& Ilias, S. Majoration de la seconde valeur propre d'un opérateur de Schrödinger sur une variété compacte et applications. J. Funct. Anal. 103 (1992), 294-316. Zbl 0766.58055 MR 1151550

7. Gonzalez, E., MASSARI, U., \& TAMAnini, I. On the regularity of boundaries of sets minimizing perimeter with a volume constraint. Indiana Univ. Math. J. 32 (1983), 25-37. Zbl 0486.49024 MR 0684753

8. Grosse-Brauckmann, K. Cousins of constant mean curvature surfaces. Global Theory of Minimal Surfaces, Clay Math. Proc. 2, Amer. Math. Soc., Providence, RI (2005), 747-767. Zbl 1110.53041 MR 2167287

9. Hauswirth, L., PÉrez, J., Romon, P., \& Ros, A. The periodic isoperimetric problem. Trans. Amer. Math. Soc. 356 (2003), 2025-2047. Zbl 1046.52002 MR 2031051

10. Hersch, J. Quatre propriétés isopérimétriques des membranes sphériques homogènes. C. R. Acad. Sci. Paris Sér. A-B 270 (1970), A1645-A1648. Zbl 0224.73083 MR 0292357

11. Lawson, H. B. Complete minimal surfaces in $S^{3}$. Ann. of Math. 92 (1970), 335-374. Zbl 0205.52001 MR 0270280

12. Lenz, P., Bechinger, C., Schäfle, C., Leiderer, P., \& Lipowsky, R. Perforated wetting layers from periodic patterns of lyophobic surface domains. Langmuir 17 (2001), 7814-7822.

13. Matsen, M. W. The standard Gaussian model for block copolymer melts. J. Phys.: Condens. Matter 14 (2002), R21-R47.

14. Meeks III, W. H. Lectures on Plateau's problem. Scola de Geometria Diferencial, Universidade Federal do Ceará (Brazil) (1978).

15. Morgan, F. Regularity of isoperimetric hypersurfaces in Riemannian manifolds. Trans. Amer. Math. Soc. 355 (2003), 5041-5052. Zbl 1063.49031 MR 1997594 
16. Palmer, B. Index and stability of harmonic Gauss maps. Math. Z. 206 (1991), 563-566. Zbl 0727.58011 MR 1100840

17. Pedrosa, R. The isoperimetric problem in spherical cylinders. Ann. Global Anal. Geom. 26 (2004), 333-354. Zbl 1082.53066 MR 2103404

18. Ritoré, M. Superficies con curvatura media constante. PhD Thesis, Granada (1994), http://www.ugr.es/ ritore.

19. Ritoré, M. Index one minimal surfaces in flat three-space forms. Indiana Univ. Math. J. 46 (1997), 1137-1153. Zbl 0922.53022 MR 1631568

20. Ritoré, M., \& Ros, A. Stable constant mean curvature tori and the isoperimetric problem in three space forms. Comment. Math. Helv. 67 (1992), 293-305. Zbl 0760.53037 MR 1161286

21. Ritoré, M., \& Ros, A. The spaces of index one minimal surfaces and stable constant mean curvature surfaces embedded in flat three manifolds. Trans. Amer. Math. Soc. 348 (1996), 391-410. Zbl 0867.53007 MR 1322955

22. ROMON, P. Personal communication.

23. Ros, A. The isoperimetric problem. Global Theory of Minimal Surfaces, Clay Math. Proc. 2, Amer. Math. Soc., Providence, RI (2005), 175-209. Zbl pre05052283 MR 2167260

24. Ros, A. Isoperimetric inequalities in crystallography. J. Amer. Math. Soc. 17 (2004), 373-388. Zbl 1048.53006 MR 2051615

25. Ros, A. One-sided complete stable minimal surfaces. J. Differential Geom. 74 (2006), 69-92. Zbl 1110.53009 MR 2260928

26. Ros, A., \& Rosenberg, H. Properly embedded surfaces with constant mean curvature. Preprint.

27. Ross, M. Schwarz' $P$ and $D$ surfaces are stable. Differential Geom. Appl. 2 (1992), 179-195. Zbl 0747.53010 MR 1245555

28. SZEGÖ, G. Inequalities for certain eigenvalues of a membrane of given area. J. Rational Mech. Anal. 3 (1954), 343-356. Zbl 0055.08802 MR 0061749

29. YANG, P., \& YAU, S. T. Eigenvalues of the Laplacian of compact Riemann surfaces and minimal submanifolds. Ann. Scuola Norm. Sup. Pisa Cl. Sci. (4) 7 (1980), 55-63. Zbl 0446.58017 MR 0577325

30. YAU, S. T. Nonlinear analysis in geometry. Enseign. Math. 33 (1987), 109-158. Zbl 0631.53002 MR 0896385 